information regarding presenting symptoms, response to pharmacological treatment, requirement for nasojejunal feeding or surgical jejunostomy and subsequent tertiary referral for consideration of gastric neurostimulation. Patients were nominally split into those with prolonged and significantly prolonged GET. Specific clinical features and subsequent response to treatment were compared. Characteristics common to both groups were identified and outcomes of atypical treatment strategies noted.

Results The cohort ( $n=21,18$ female, 3 male, ages 18-74) was categorised on the basis of GET (Measured on nuclear study. Prolonged: $n=13$, GET $<130$ min. Significantly prolonged: $n=8$, GET $>130 \mathrm{~min}$ ). Whereas prevalence of most presenting symptoms was similar between groups weight loss and constipation were seen only in the significantly prolonged group (50\% in both instances). Significant clinical improvement with oral domperidone and metoclopramide was disappointingly unusual, however was slightly more common in the prolonged group (43\% vs $25 \%$ ). GET was not a useful predictor of treatment response. Of four patients requiring jejunal feeding and tertiary referral for neurostimulation, two had only marginal prolongation in GET. Of four patients started on the 5HT4 agonist prucalopride for concomitant constipation, three noticed incidental improvement in gastroparetic symptoms. One patient reported improvement following Chinese acupuncture.

Conclusion The case series illustrates inherent difficulties in diagnosing and managing gastroparesis. It notes certain clinical features, such as weight loss and constipation, which may relate to more significant prolongations in GET. In isolation GET appeared to be a poor marker of disease severity and was not useful in reliably predicting treatment response or requirement for subsequent tertiary referral. Observations of symptomatic improvement with prucalopride, a drug currently unlicensed for this indication, suggest further study into its wider therapeutic use should be afforded.

Competing interests B Hudson: None declared, F Fayyaz: None declared, R Makins Conflict with: This author has previously acted as an advisor for shire pharmaceuticals.

\section{PWE-046 THE DEVELOPMENT OF HUMAN ESOPHAGEAL PAIN HYPERSENSITIVITY IS ASSOCIATED WITH ANXIETY AND SYMPATHETIC NERVOUS SYSTEM ACTIVATION}

doi:10.1136/gutjnl-2012-302514d.46

${ }^{1} \mathrm{C}$ A Botha, ${ }^{*} \mathrm{C} \mathrm{C}$ Knowels, ${ }^{1} \mathrm{C}$ Aziz. ${ }^{1}$ Wingate Institute, Barts and The London School of Medicine and Dentistry, Queen Mary University of London, UK; ${ }^{2}$ Academic Surgery, Barts and The London School of Medicine and Dentistry, Queen Mary University of London, London, UK

Introduction Experimental acid infusion in the distal oesophagus leads to secondary hyperalgesia in the proximal oesophagus in most healthy subjects but $30 \%$ remain resistant. The factors that mediate differences in sensitisation to acid are unclear and their study may help to understand risk factors for esophageal hypersensitivity in gastro-esophageal reflux disease. We aimed to determine the psychophysiological factors which predict the development of esophageal pain hypersensitivity (EPH) to acid infusion in healthy subjects.

Methods 52 healthy volunteers (mean age 29 yrs, range $19-49$ yrs; 33 male) underwent psychological profiling for, anxiety, depression and personality type. Baseline pain thresholds (PT) to proximal esophageal electrical stimulation were measured before hydrochloric acid infusion $(0.15 \mathrm{M})$ in the distal oesophagus for $30 \mathrm{~min}$. This was followed by esophageal pain threshold measurements using visual analogue scales to electrical stimulation in the proximal, unexposed oesophagus at 30,60 and $90 \mathrm{~min}$. Parasympathetic (Cardiac Vagal Tone-CVT) and Sympathetic Nervous System (RR interval) responses were monitored throughout the study. Volunteers were classified as sensitisers if the proximal PT fell $\geq 6 \mathrm{~mA}$ after esophageal acidification.
Results Four subjects dropped out, 35 subjects (73\%) sensitised to acid, and 13 subjects $(27 \%)$ did not sensitise. There was no difference in the age, sex or in personality domains between sensitisers and non-sensitisers $(p>0.05)$. Spielberg trait anxiety comparison indicated a trend, $\mathrm{p}=0.08$ for sensitisers to be more anxious than non-sensitisers. At baseline there was no difference in CVT and RR interval between the two groups. During acid infusion the key difference in autonomic nerves system response was that in the sensitisers the RR interval was shorter in sensitisers than nonsensitisers (sensitisers: $\Delta 1.7 \mathrm{SE} \pm 48.9$ vs non-sensitisers: $\Delta 66.1$ $\mathrm{SE} \pm 25.65 . \Delta \mathrm{RR}$ interval difference $64.4 \mathrm{~ms}(\mathrm{SE} \pm 37.3) \mathrm{p}=0.002)$. Trait anxiety demonstrated a negative correlation with the RR interval $r=-0.35(p=0.04)$

Conclusion There was a trend for sensitisers to be more anxious and at baseline there were no differences in the PNS and SNS parameters between the two groups. During acid infusion, the sensitisers had a lower RR interval than non-sensitisers suggesting a higher heart rate and therefore SNS tone. Trait anxiety showed a negative correlation with the RR interval suggesting those with higher anxiety had higher heart rate. Our results suggest that higher anxiety and SNS activation has a pro nociceptive effect on the development of postacid infusion esophageal sensitisation. This may explain why individuals with anxiety or stress at the time of visceral injury or inflammation are more likely to go onto develop EPH.

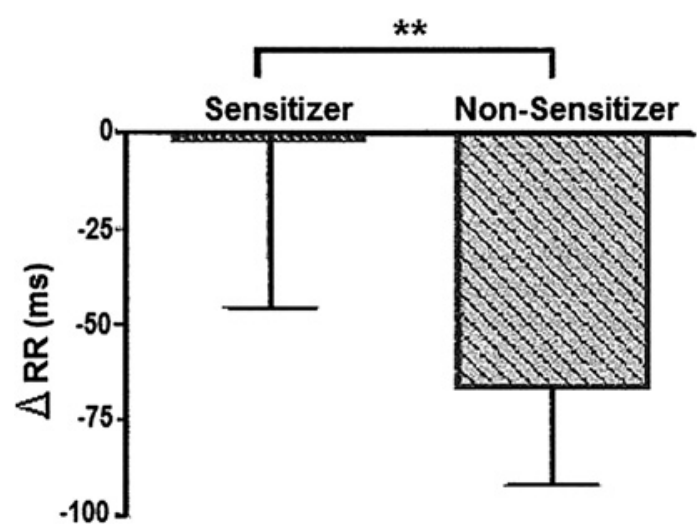

Abstract PWE-046 Figure 1 Mean difference in $\triangle R R$ interval. ${ }^{* *} 64.468 \mathrm{~ms}$ (SE \pm 37.305$) \mathrm{p}=0.0029$.

Competing interests None declared.

\section{PWE-047 FULL THICKNESS SMALL BOWEL BIOPSY IN GUT DYSMOTILITY. CASE SERIES OF EIGHT PATIENTS ILLUSTRATING THE BENEFITS AS A DIAGNOSTIC TOOL}

doi:10.1136/gutjnl-2012-302514d.47

${ }^{1}$ E Harrison, * ${ }^{2} \mathrm{~J}$ E Martin, ${ }^{1} \mathrm{~S}$ C Cooper. ${ }^{1}$ Department of Gastroenterology, Dudley Group of Hospitals NHS Foundation Trust, Birmingham, UK; ${ }^{2}$ Blizard Institute of Cell and Molecular Science Pathology Group, The Royal London Hospital, London, UK

Introduction Full thickness small bowel biopsies offer the opportunity to make a definitive diagnosis in patients with gastrointestinal dysmotility. However, our experience suggests that standard histological reporting may fail to achieve this diagnosis. This study compares local diagnoses to those of a national expert. Methods We retrospectively reviewed eight sequential patients with symptomatic gastrointestinal dysmotility who were reviewed at a tertiary centre having had locally reported full thickness small bowel biopsies. Local histological reports were compared to those of a national expert. Patient notes were reviewed to determine the clinical impact of achieving a definitive diagnosis. Some patients were questioned regarding the impact of their definitive diagnosis. 
Results The biopsies of the eight sequential patients had been reported by one of four centres within the West Midlands. All, except one, of these local reports were normal. However, subsequent review of all eight biopsies by the national expert identified pathological changes in all. Diagnoses established included two cases of polyglucosan body myopathy, confirmation of NSAID enteropathy and differing forms of inflammation (eg, lymphocytic plexitis). Establishing these diagnoses enabled accurate prognoses and implementation of subsequent management, including continuation of home parenteral nutrition (HPN, $\mathrm{n}=6$ ) and consideration for small intestinal transplantation (SIT, $n=3)$. Patients questioned reported additional benefits.

Conclusion Gut dysmotility can be highly symptomatic and debilitating leading to intestinal failure (IF), HPN and SIT. Clinical decisions for consideration for HPN and SIT are complex. Decisions must consider the potential for morbidity and mortality against the potential for improvement in nutritional status, quality of life and survival. A full thickness small bowel biopsy, while invasive, offers opportunity for a definitive diagnosis, and thus a prognosis. Published series report an $81 \%$ diagnostic yield for small bowel biopsies in patients with suspected gastrointestinal neuromuscular disorders, when using routine and immunohisto-chemical techniques. ${ }^{1}$ However, standard histopathological reporting, which is often based on H\&E staining alone, has less potential for achieving a diagnosis. This is shown by our study in which a diagnosis was achieved in only $13 \%$. Thus, our study highlights the importance of expert review and demonstrates the importance of achieving a diagnosis for patient and clinician.

Competing interests None declared.

\section{REFERENCE}

1. Knowles CH, Veress B, Tornblom $\mathrm{H}$, et al. Safety and diagnostic yield of laparoscopically assisted full-thickness bowel biopsy. Neurogastroenterol Motil 2008;20:774-9. gastric content volume were analysed: Gastric contents volume after meal ingestion (GCV0), GE half-time [T50], and GErate@T50 $[\mathrm{ml} / \mathrm{min}]$.

Results FDs ( $\mathrm{n}=8 ; 7$ female) were each compared to those of three matched HVs $(n=24)$. HVs weighed more than FDs $(p<0.018)$ fullness at $400 \mathrm{ml}$ was similar $(p=0.21)$ but dyspeptic sensations were lower (bloating, nausea, pain, $\mathrm{p}<0.01$ ) and MTV was greater (median 960 (IOR 750-1330) vs $480( \pm 400-760) \mathrm{ml}, \mathrm{p}=0.015)$. With GS, HVs had higher GCV0 than FDs (345 (333-358) vs 325 (310-350) $\mathrm{ml} ; \mathrm{p}=0.052)$, T50 (48 (39-56) vs 52 (44-54) min; $\mathrm{p}=0.710)$ was similar but GErate@T50 was faster $(3.5(3.0-4.2) \mathrm{ml} /$ min vs $2.7(2.1-3.1) \mathrm{ml} / \mathrm{min}$; $\mathrm{p}=0.012)$. With MRI, compared to GS, measurements of GCV and T50 were larger $(p<0.001)$, and GErate was slower $(p=0.012)$; but no significant differences between groups.

Conclusion FD patients are characterised by abnormal gastric sensorimotor response to a large, liquid nutrient meal. Rapid early emptying (reduced GCV0) is followed by slow late emptying (slow GErate@T50). These GS measurements, with dyspeptic symptoms at $400 \mathrm{ml}$, are biomarkers in FD. MRI measurements of GCV, residual volume, meal and secretions do not provide the same clarity. These findings are consistent with the hypothesis that impaired accommodation in gastric filling in FD leads to rapid nutrient delivery to small bowel triggering powerful neuro-hormonal feedback that slows subsequent emptying.

Competing interests None declared.

\section{PWE-049 EFFECTS OF AGE, SEX AND OBESITY ON SATIATION ASSESSED BY NUTRIENT DRINK TEST AND GASTRIC EMPTYING (GE) ASSESSED BY NON-INVASIVE GASTRIC SCINTIGRAPHY (GS) AND MRI: ANALYSIS AND COMPARISON OF METHODS}

doi:10.1136/gutjin-2012-302514d.49

${ }^{1} \mathrm{H}$ Parker, ${ }^{*}{ }^{2} \mathrm{C}$ Hoad, ${ }^{1} \mathrm{~N}$ Hudders, ${ }^{1} \mathrm{E}$ Tucker, ${ }^{3} \mathrm{~A}$ Perkins, ${ }^{3} \mathrm{P}$ E Blackshaw, ${ }^{1} \mathrm{~L}$ Marciani, ${ }^{2} \mathrm{C}$ Costigan, ${ }^{2} \mathrm{P}$ Gowland, ${ }^{1} \mathrm{M}$ Fox. ${ }^{1} \mathrm{~N} / \mathrm{H} R$ Biomedical Research Unit, University of Nottingham, Nottingham, UK; ${ }^{2}$ Sir Peter Mansfield Magnetic Resonance Centre, University of Nottingham, Nottingham, UK; ${ }^{3}$ Division of Radiology and Imaging Sciences, University of Nottingham, Nottingham, UK DYSPEPSIA AND HEALTH ASSESSED BY NON-INVASIVE GASTRIC SCINTIGRAPHY (GS) AND MRI: A PILOT STUDY TO IDENTIFY CANDIDATE BIOMARKERS

doi:10.1136/gutjnl-2012-302514d.48

${ }^{1} \mathrm{E}$ Tucker, ${ }^{*}{ }^{1} \mathrm{H}$ Parker, ${ }^{2} \mathrm{C}$ Hoad, ${ }^{1} \mathrm{~N}$ Hudders, ${ }^{3} \mathrm{~A}$ Perkins, ${ }^{3} \mathrm{P}$ E Blackshaw, ${ }^{1} \mathrm{~L}$ Marciani, ${ }^{2} \mathrm{C}$ Costigan, ${ }^{2} \mathrm{P}$ Gowland, ${ }^{1} \mathrm{M}$ Fox. ${ }^{1} \mathrm{~N}$ IHR Biomedical Research Unit, University of Nottingham, Nottingham, UK; ${ }^{2}$ Sir Peter Mansfield Magnetic Resonance Centre, University of Nottingham, Nottingham, UK; ${ }^{3}$ Division of Radiology and Imaging Sciences, University of Nottingham, Nottingham, UK

Introduction Dyspeptic symptoms are common but investigations rarely explain them. This lack of information may be because: (1) Current test meals are small ( $\sim 200 \mathrm{ml})$ and don't trigger symptoms (2) gastric emptying half time (T50) and/or retention at 2 or $4 \mathrm{~h}$ may not elicit underlying pathophysiology. By contrast, MRI studies suggest that gastric volume change after a meal may identify impaired accommodation in functional dyspepsia (FDs).

Aim GS and MRI with a $400 \mathrm{ml}$ liquid test meal were applied to identify candidate biomarkers that distinguish FDs from healthy volunteers (HVs).

Methods FDs with postprandial distress by Rome III criteria and normal endoscopy or $24 \mathrm{~h} \mathrm{pH}$-studies were recruited. Results were compared to age and sex matched HVs. Sensation at $400 \mathrm{ml}$ and Maximum Tolerated Volume (MTV) was assessed by nutrient drink test $(0.75 \mathrm{kcal} / \mathrm{ml} @ 40 \mathrm{ml} / \mathrm{min})$. Participants were then randomised to GS and MRI with $400 \mathrm{ml}$ liquid test meal (0.75 kcal/ml@40 ml/ $\mathrm{min}$ ) on two separate days. Directly comparable measurements of
Introduction The relationship of demographic and anthropometric factors on satiation and gastric functions is incompletely understood. Optimal methodology to assess meal intake factors and postprandial GE has not been established. Current test meals are small and may not be sufficient to assess satiation and postprandial responses. GS measures gastric meal retention only. MRI measures gastric content volume (meal and secretions)

Aim This study assessed the effects of age, sex and obesity on maximum tolerated volume (MTV) assessed by nutrient drink test (NDT) in healthy volunteers (HVs). GS and MRI assessed gastric function and GE after ingestion of a $400 \mathrm{ml}$ liquid test meal (same nutrient drink) that triggered sensation of fullness in $>90 \% \mathrm{HVs}$.

Methods Adult HVs were recruited and stratified by sex and age aiming to study 10 men and women in age groups $<40,40-60$, $>60$ yrs. Exclusions included history of GI surgery and obesity $\left(\mathrm{BMI}>30 \mathrm{~kg} / \mathrm{m}^{2}\right)$. MTV was assessed at screening by NDT $(0.75 \mathrm{kcal} / \mathrm{ml}$ at $40 \mathrm{ml} / \mathrm{min})$, all $\mathrm{HVs}$ ingested $>400 \mathrm{ml}$. Eligible participants were randomised to gastric imaging by GS and MRI on two separate days. HVs ingested $400 \mathrm{ml}$ liquid nutrient at $40 \mathrm{ml} /$ min. Gastric content volume was monitored over $4 \mathrm{~h}$. GS and MRI measured GE half-time [T50], GErate maximum and GErate@T50 $[\mathrm{ml} / \mathrm{min}]$. Univariate and multiple linear regression models assessed the effects of demographic and anthropometric parameters on gastric function. associated with male sex $\left(R^{2}=20 \%, p<0.001\right)$, height $\left(R^{2}=9 \%\right.$,
Results $53 \mathrm{HV}$ s completed the study (6-10 in each group). MTV 